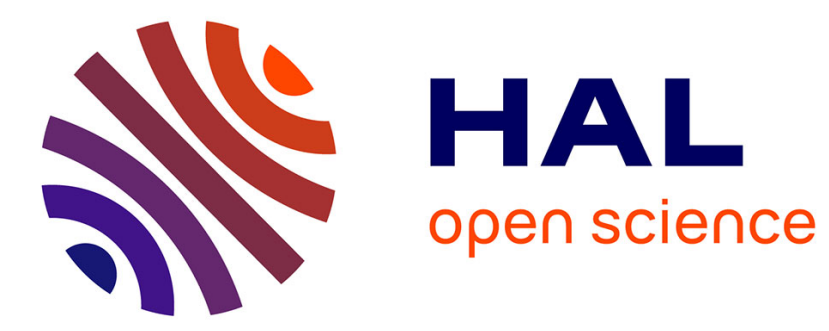

\title{
Radiative transfer with partial coherence in optically thick plasmas \\ Joël Rosato
}

\section{To cite this version:}

Joël Rosato. Radiative transfer with partial coherence in optically thick plasmas. Physical Review E : Statistical, Nonlinear, and Soft Matter Physics, 2013, pp.DOI: 10.1103/PhysRevE.87.043108. hal00830443

\section{HAL Id: hal-00830443 \\ https://hal.science/hal-00830443}

Submitted on 5 Jun 2013

HAL is a multi-disciplinary open access archive for the deposit and dissemination of scientific research documents, whether they are published or not. The documents may come from teaching and research institutions in France or abroad, or from public or private research centers.
L'archive ouverte pluridisciplinaire HAL, est destinée au dépôt et à la diffusion de documents scientifiques de niveau recherche, publiés ou non, émanant des établissements d'enseignement et de recherche français ou étrangers, des laboratoires publics ou privés. 


\title{
Radiative transfer with partial coherence in optically thick plasmas
}

\author{
J. Rosato* \\ Aix-Marseille Université, CNRS, PIIM UMR 7345, F-13397 Marseille Cedex 20, France
}

\begin{abstract}
A quantum transport model for atomic line radiation in plasmas is developed and analyzed. It is found that the Wigner phase space formulation of QED provides a consistent way to address the wave-particle duality in radiative transfer problems. If the photons' thermal de Broglie length is much smaller than all of the spatial scales of the problem under consideration (large spectral band limit), the radiation is not coherent and radiative transfer can be addressed with usual treatments. In the general case, the Heisenberg uncertainty relation yields ambiguities in the description of the radiation-matter interaction mechanisms. We examine this issue and show that an accurate description of radiative transfer should involve a model with nonlocal interactions, and requires an appropriate coarse-graining procedure. Calculations of transmission factors and absorption spectra in ideal cases are performed, and indicate that significant misinterpretations can be done in spectroscopic diagnostics if the radiation coherence is not well accounted for. Applications to laser physics are also discussed.
\end{abstract}

PACS numbers: $05.60 . \mathrm{Gg}, 52.25 . \mathrm{Os}, 42.25 . \mathrm{Kb}, 32.70 . \mathrm{Jz}$

\section{INTRODUCTION}

The usual approach to address radiative transfer problems involves a transport equation of Boltzmann-type, referred to as "radiative transfer equation", accounting for radiation-matter interaction processes such as spontaneous/stimulated emission and absorption [1,2]. The most natural domain of application is astrophysics [1], where information on the objects can only be obtained from passive observation of the emitted radiation. The large size of galaxies, stellar atmospheres or nebula leads to the presence of reabsorption effects and makes the use of a transport theory for photons unavoidable. Opacity models for radiative transfer are widely used in highenergy density physics, in astrophysics (e.g. in the core of stars) but also in the framework of fusion research, e.g., to characterize the warm and hot dense matter present in imploding inertial confinement capsules [3-7]. Another important application of opacity models is provided by spectroscopic diagnostics and radiation transport simulations in magnetic fusion, in particular with the preparation of ITER (currently under construction in France) and the development of integrated modeling codes supporting its operation [8-14]. Applications can be found in other contexts, e.g., in investigations of magneto-optical traps ("photon bubbles" [15]), gas discharge lamps [16], biology [17]. As a rule, reliability in the interpretation of spectra, as well as in predictions, relies on the development of accurate spectroscopy models accounting for opacity effects.

Although the particle ("photon") picture of radiation transport is suitable for numerical simulations (e.g. based on Monte-Carlo procedure $[9,18]$ ), it may be inaccurate if the light's coherence length $\lambda^{2} / \Delta \lambda$ (with $\lambda, \Delta \lambda$ being the characteristic wavelength of the radiation and

*Electronic address: joel.rosato@univ-amu.fr its spectral width, respectively) [19] is significant with respect to the spatial scales of interest of the system under consideration. This concerns atomic spectral lines in particular given the largeness of $\lambda / \Delta \lambda$, by definition. An interpretation of this inaccuracy is provided by the correspondence between the coherence length and the photons' thermal de Broglie length $\hbar / \Delta p$ for radiation with the typical momentum detuning $\Delta p$. The Heisenberg uncertainty principle prevents one from localizing light quanta in a volume smaller than $(\hbar / \Delta p)^{3}$, in contrast with the predictions of the standard radiative transfer theory, which is essentially local in space. A quantum version of the radiative transfer equation, accounting for coherence, cannot be obtained from direct application of the correspondence principle because the photon as viewed from QED does not have a definite position operator due to symmetry considerations [20-24] (see also [25-28] for recent works). A more convenient approach is provided by the quantum phase space formalism adapted to second quantization (e.g. [29-34] and Refs. therein). The quantum phase space formalism has been introduced in the seminal article by Wigner more than eighty years ago [35] and has found applications in many domains, including collision theory [36], solid state physics [37], general transport phenomena [38], and also fundamental quantum mechanics ("phase space formulation" [39]; e.g. $[40,41]$ for recent applications). The quantum phase space formalism is also used in optics, e.g. for the modeling of the propagation of fields [42]. However, in spite of a rich literature on the subject there has been only a few explicit applications to problems involving radiation transport. Recently [43-45], a quantum transport model for photons that accounts for spatial coherence has been derived and applications to ideal systems have indicated an alteration of the one-photon Wigner function in cases where the coherence length is comparable either to the photon mean free path or to the characteristic size of the system. Although more general than the standard radiative transfer theory, this model has no 
obvious interpretation. The transport equation involves source and loss terms delocalized through a phase space integral that cannot be reduced to simple convolution, which makes standard treatments based on Fourier analysis tricky. On the other hand, the presence of complex line shape functions raises a problem of consistency in the probabilistic interpretation of the emission and absorption processes. The aim of this paper is to provide an analysis of these issues and to illustrate the applicability of the model in realistic cases. A theoretical deduction of the transport equation, based on the previous works [43-45], is presented. Our development involves a master equation for photons and uses concepts of the theory of open quantum systems. The master equation is derived in Sec. II, and it is adapted to the quantum phase space formalism in Sec. III. It is shown that accounting for coherence effects amounts to write down a master equation of Lindblad-type and to keep non-diagonal terms of the radiation density matrix in the Fock states. Section IV addresses the link between Wigner functions and observable quantities. In Sec. V, the transport of atomic excitation is examined within a generalization of the HolsteinBiberman theory. A simplification the transport model, based on a series expansion in powers of $\hbar$, is examined in Sec. VI. Absorption lines in a slab geometry are considered as an example of application. Finally, applications to laser physics are discussed in Sec. VII.

\section{MASTER EQUATION FOR THE PHOTONS}

The development presented hereafter concerns spectral lines in particular, given the largeness of $\lambda / \Delta \lambda$. The emitters and absorbers can be either neutral atoms or multicharged ions. Because of their formal similarity (in terms of energy levels), these species will simply be referred to as "atoms". The system of interest is a gas of atoms interacting with the radiation field described within the second quantization. The standard discretization procedure is adopted [46]: we take an infinite set of quantum harmonic oscillators, each mode is labeled by the wavevector $\mathbf{k}_{j}=2 \pi \mathbf{n}_{j} / L$ ( $\mathbf{n}_{j}$ is a triplet of integers) and the polarization vector $\varepsilon_{j}$, and the quantization is done in a cubic box with periodic boundary conditions. The formal developments are done keeping the size $L$ of the cube finite, and then taking the limit $L \rightarrow \infty$. We denote $a_{j}$ the photon annihilation operator, $a_{j}^{\dagger}$ the creation operator, and $N_{j}=a_{j}^{\dagger} a_{j}$ the number operator. The annihilation and creation operators satisfy the commutation rules $\left[a_{j}, a_{j^{\prime}}^{\dagger}\right]=\delta_{j j^{\prime}}$ and $\left[a_{j}, a_{j^{\prime}}\right]=0=\left[a_{j}^{\dagger}, a_{j^{\prime}}^{\dagger}\right]$. The quantized electric and magnetic field are described in terms of the creation and annihilation operators as

$$
\mathbf{E}(\mathbf{r})=\sum_{j} i \sqrt{\frac{\hbar \omega_{j}}{2 \varepsilon_{0} L^{3}}}\left(a_{j} e^{i \mathbf{k}_{j} \cdot \mathbf{r}}-a_{j}^{\dagger} e^{-i \mathbf{k}_{j} \cdot \mathbf{r}}\right) \varepsilon_{j}
$$

$$
\mathbf{B}(\mathbf{r})=\sum_{j} i \sqrt{\frac{\hbar}{2 \varepsilon_{0} \omega_{j} L^{3}}}\left(a_{j} e^{i \mathbf{k}_{j} \cdot \mathbf{r}}-a_{j}^{\dagger} e^{-i \mathbf{k}_{j} \cdot \mathbf{r}}\right) \mathbf{k}_{j} \times \varepsilon_{j},
$$

where $\omega_{j}=c k_{j}$ is the frequency corresponding to mode $j$. The Hamiltonian of the free radiation field $H_{R}$ is given by

$$
H_{R}=\sum_{j} \hbar \omega_{j}\left(N_{j}+\frac{1}{2}\right) .
$$

Its eigenvectors are elements of the Fock space formed by the kets $\left|n_{1} \ldots n_{p} \ldots\right\rangle$. Each ket $\left|0_{1} \ldots 0_{p-1}, n_{p}, 0_{p+1} \ldots\right\rangle \equiv\left|n_{p}\right\rangle$ denotes a state with $n_{p}$ photons of momentum $\mathbf{p}_{p}=\hbar \mathbf{k}_{p}$ and polarization $\varepsilon_{p}$. The state with no photons $\left|0_{1} \ldots 0_{p} \ldots\right\rangle \equiv|0\rangle$ is the vacuum state. The Hamiltonian contains additional terms $H_{A}$ and $V$, which account for the dynamics of the atoms and their interaction with the radiation field, respectively. In the following we consider bound-bound transitions and describe the interaction term within the dipole approximation:

$$
V=\sum_{a}-\mathbf{d}_{a} \cdot \mathbf{E}\left(\mathbf{r}_{a}\right) .
$$

Here, $\mathbf{d}_{a}$ and $\mathbf{r}_{a}$ are respectively the dipole operator and position operator of the center of mass of the ath atom. We assume that the radiation and matter are weakly coupled, so that the evolution of the density operator can be described using a quantum master equation. Following standard approaches [47-49], we write

$$
\begin{aligned}
\frac{d \rho_{R}}{d t}(t)= & -\frac{i}{\hbar}\left[H_{R}, \rho_{R}(t)\right] \\
& -\frac{1}{\hbar^{2}} \int_{0}^{\infty} d \tau \operatorname{Tr}_{A}[V,[\tilde{V}(-\tau), \rho(t)]],
\end{aligned}
$$

where $\rho_{R}=\operatorname{Tr}_{A}(\rho)$ denotes the density operator of the radiation, obtained from partial trace of the whole system's density operator $\rho$ over the atomic states, and $\tilde{V}(-\tau)=e^{-i H_{0} \tau / \hbar} V e^{i H_{0} \tau / \hbar}$ is the interaction taken in the interaction picture with $H_{0}=H_{A}+H_{R}$. The weak coupling approximation yields the factorization $\rho=\rho_{A} \rho_{R}$ in the second term of the right-hand side with $\rho_{A}=\operatorname{Tr}_{R}(\rho)$. Equation (5) can be written in a form similar to the Lindblad equation, by using techniques involved in the theory of open quantum systems [50]. We expand the commutator and write it as a quadratic function of the creation and annihilation operators. The terms proportional to $\rho_{R} a_{j} a_{j^{\prime}}, \rho_{R} a_{j}^{\dagger} a_{j^{\prime}}^{\dagger}$, and their permutations, are neglected since they are not resonant (this assumption is similar to the rotating wave approximation [32]). Algebraic manipulations yield the following expression

$$
\begin{aligned}
- & \frac{1}{\hbar^{2}} \int_{0}^{\infty} d \tau \operatorname{Tr}_{A}[V,[\tilde{V}(-\tau), \rho(t)]] \\
= & \sum_{j j^{\prime}}\left[\Gamma_{1 j j^{\prime}}^{*} a_{j} \rho_{R} a_{j^{\prime}}^{\dagger}+\Gamma_{2 j j^{\prime}}^{*} a_{j}^{\dagger} \rho_{R} a_{j^{\prime}}\right. \\
& \left.-\Gamma_{1 j j^{\prime}} a_{j}^{\dagger} a_{j^{\prime}} \rho_{R}-\Gamma_{2 j j^{\prime}} a_{j} a_{j^{\prime}}^{\dagger} \rho_{R}\right]+ \text { H.c. },
\end{aligned}
$$


where H.c. stands for Hermitian conjugate and $\Gamma_{1 j j^{\prime}}$, $\Gamma_{2 j j^{\prime}}$ are two scalars that depend on the atomic variables only. They are defined as half-Fourier transforms of correlation functions

$$
\begin{gathered}
\Gamma_{1 j j^{\prime}}=\frac{\sqrt{\omega_{j} \omega_{j^{\prime}}}}{2 \varepsilon_{0} \hbar L^{3}} \sum_{a a^{\prime}} \int_{0}^{\infty} d \tau\left\langle D_{a j} \tilde{D}_{a^{\prime} j^{\prime}}^{\dagger}(-\tau)\right\rangle e^{i \omega_{j^{\prime}} \tau}, \\
\Gamma_{2 j j^{\prime}}=\frac{\sqrt{\omega_{j} \omega_{j^{\prime}}}}{2 \varepsilon_{0} \hbar L^{3}} \sum_{a a^{\prime}} \int_{0}^{\infty} d \tau\left\langle D_{a j}^{\dagger} \tilde{D}_{a^{\prime} j^{\prime}}(-\tau)\right\rangle e^{-i \omega_{j^{\prime}} \tau},
\end{gathered}
$$

and they are related to the spectral profile of the emission and absorption processes, as will be shown below. $D_{a j}=\mathbf{d}_{a} \cdot \varepsilon_{j} \exp \left(-i \mathbf{k}_{j} \cdot \mathbf{r}_{a}\right)$ is a shortcut notation; the exponential stems from the decomposition of the electric field given in Eq. (1). The brackets $\langle\ldots\rangle$ denote a statistical average performed over the atomic states, internal and external. If we assume independent atoms, with independent external and internal states, moving classically along straight lines during the characteristic correlation time, the averages are factorized and the $\Gamma \mathrm{s}$ become simpler

$$
\begin{aligned}
& \Gamma_{1 j j^{\prime}}=\frac{\sqrt{\omega_{j} \omega_{j^{\prime}}} \mathcal{N}_{\mathrm{at}}}{2 \varepsilon_{0} \hbar L^{3}} \int_{0}^{\infty} d \tau\left\langle\mathbf{d} \cdot \boldsymbol{\varepsilon}_{j} \tilde{\mathbf{d}}(-\tau) \cdot \boldsymbol{\varepsilon}_{j^{\prime}}\right\rangle \\
& \times\left\langle e^{-i \mathbf{k}_{j j^{\prime}} \cdot \mathbf{r}} e^{i\left(\omega_{j^{\prime}}-\mathbf{k}_{j^{\prime}} \cdot \mathbf{v}\right) \tau}\right\rangle, \\
& \Gamma_{2 j j^{\prime}}=\frac{\sqrt{\omega_{j} \omega_{j^{\prime}}} \mathcal{N}_{\mathrm{at}}}{2 \varepsilon_{0} \hbar L^{3}} \int_{0}^{\infty} d \tau\left\langle\mathbf{d} \cdot \boldsymbol{\varepsilon}_{j} \tilde{\mathbf{d}}(-\tau) \cdot \boldsymbol{\varepsilon}_{j^{\prime}}\right\rangle \\
& \times\left\langle e^{-i \mathbf{k}_{j j^{\prime}} \cdot \mathbf{r}} e^{i\left(\omega_{j^{\prime}}-\mathbf{k}_{j^{\prime}} \cdot \mathbf{v}\right) \tau}\right\rangle^{*} .
\end{aligned}
$$

Here, the indices $a, a^{\prime}$ have been removed and the sums have been replaced by multiplication with the number of atoms $\mathcal{N}_{\text {at }}$, in agreement with the independent atoms assumption. The first average is performed over the internal atomic states and provides the dipole autocorrelation function. In the following we assume unpolarized radiation, so that $\left\langle\mathbf{d} \cdot \varepsilon_{j} \tilde{\mathbf{d}}(-\tau) \cdot \varepsilon_{j^{\prime}}\right\rangle$ can be replaced by $\left\langle\mathbf{d} \cdot \boldsymbol{\varepsilon}_{j} \tilde{\mathbf{d}}(-\tau) \cdot \boldsymbol{\varepsilon}_{j}\right\rangle \delta_{\boldsymbol{\varepsilon}_{j} \boldsymbol{\varepsilon}_{j^{\prime}}}$, with $\delta_{\boldsymbol{\varepsilon}_{j} \boldsymbol{\varepsilon}_{j^{\prime}}}$ being the Kronecker symbol. The second average is performed using the atoms' one-particle phase space distribution. In the exponential, the notation $\mathbf{k}_{j j^{\prime}}=\mathbf{k}_{j}-\mathbf{k}_{j^{\prime}}$ is used. It is convenient to introduce the following notations:

$$
\begin{gathered}
\chi_{\mathrm{c}, \mathrm{abs}}(\mathbf{r}, \mathbf{p}, t)=\frac{\hbar \omega_{0}}{4 \pi} B_{g e} N_{g}(\mathbf{r}, t) \phi_{c}(\omega, \hat{\mathbf{n}}, \mathbf{r}, t), \\
\chi_{\mathrm{c}, \mathrm{em}}(\mathbf{r}, \mathbf{p}, t)=\frac{\hbar \omega_{0}}{4 \pi} B_{e g} N_{e}(\mathbf{r}, t) \phi_{c}(\omega, \hat{\mathbf{n}}, \mathbf{r}, t), \\
\chi_{c}=\chi_{\mathrm{c}, \mathrm{abs}}-\chi_{\mathrm{c}, \mathrm{em} .}
\end{gathered}
$$

In Eqs. (11), (12), $N_{g}(\mathbf{r}, t)$ and $N_{e}(\mathbf{r}, t)$ denote the population densities of the lower $(g)$ and upper $(e)$ levels of the transition at location $\mathbf{r}$ and time $t, B_{g e}$ and $B_{e g}$ are the Einstein coefficients for absorption and stimulated emission, and $\hbar \omega_{0}$ is the corresponding energy. The quantity $\phi_{c}$ denotes the complex spectral line shape function, with $\omega=|\mathbf{p}| c / \hbar$ and $\hat{\mathbf{n}}=\mathbf{p} /|\mathbf{p}|$ being the frequency and the normal unit vector corresponding to the photon momentum $\mathbf{p}$, respectively. The complex line shape function is normalized over the frequencies, i.e., $\int d \omega \int(d \Omega / 4 \pi) \phi_{c}(\omega, \hat{\mathbf{n}}, \mathbf{r}, t)=1$. Using the same notation as in [44], we write it as follows

$$
\phi_{c}(\omega, \hat{\mathbf{n}}, \mathbf{r}, t)=\int d^{3} v f(\mathbf{v} ; \mathbf{r}, t) \phi_{0 c}(\omega(1-\hat{\mathbf{n}} \cdot \mathbf{v} / c), \hat{\mathbf{n}}, \mathbf{r}, t),
$$

$$
\phi_{0 c}(\omega, \hat{\mathbf{n}}, \mathbf{r}, t)=\frac{1}{\pi} \int_{0}^{\infty} d \tau C(\tau ; \hat{\mathbf{n}}, \mathbf{r}, t) e^{-i \omega \tau},
$$

where $f$ is the atoms velocity distribution function that accounts for thermal Doppler broadening and $C$ is the autocorrelation function of the atomic dipole projected onto the polarization plane in reduced units. The quantity $\chi_{c}$ defined in Eq. (13) has the dimensions of an inverse length and denotes a complex extinction coefficient. Equations (11) and (12) allows one to write the $\Gamma \mathrm{s}$ in a more compact form

$$
\begin{gathered}
\Gamma_{1 j j^{\prime}}=\frac{c \delta_{\boldsymbol{\varepsilon}_{j} \boldsymbol{\varepsilon}_{j^{\prime}}}}{2 L^{3}} \int d^{3} r e^{-i \mathbf{k}_{j j^{\prime}} \cdot \mathbf{r}} \chi_{\mathrm{c}, \mathrm{abs}}^{*}\left(\mathbf{r}, \hbar \mathbf{k}_{j^{\prime}}\right), \\
\Gamma_{2 j j^{\prime}}=\frac{c \delta_{\boldsymbol{\varepsilon}_{j} \boldsymbol{\varepsilon}_{j^{\prime}}}}{2 L^{3}} \int d^{3} r e^{i \mathbf{k}_{j j^{\prime}} \cdot \mathbf{r}} \chi_{\mathrm{c}, \mathrm{em}}\left(\mathbf{r}, \hbar \mathbf{k}_{j^{\prime}}\right) .
\end{gathered}
$$

Here the time dependence has not been written explicitly for the sake of simplicity. Algebraic manipulations with Eqs. (5) and (6) yield an equation of Lindblad-type for the density operator $\rho_{R}$ :

$$
\frac{d \rho_{R}}{d t}(t)=-\frac{i}{\hbar}\left[H_{R}+H_{S}, \rho_{R}(t)\right]+\mathcal{D}\left(\rho_{R}(t)\right) .
$$

The shift is given by

$$
H_{S}=\sum_{j j^{\prime}}\left[S_{1 j j^{\prime}} a_{j}^{\dagger} a_{j^{\prime}}+S_{2 j j^{\prime}} a_{j} a_{j^{\prime}}^{\dagger}\right],
$$

and the dissipator reads

$$
\begin{aligned}
\mathcal{D}\left(\rho_{R}\right) & =\sum_{j j^{\prime}} \gamma_{1 j j^{\prime}}\left[a_{j^{\prime}} \rho_{R} a_{j}^{\dagger}-\frac{1}{2}\left\{a_{j}^{\dagger} a_{j^{\prime}}, \rho_{R}\right\}\right] \\
& +\sum_{j j^{\prime}} \gamma_{2 j j^{\prime}}\left[a_{j^{\prime}}^{\dagger} \rho_{R} a_{j}-\frac{1}{2}\left\{a_{j} a_{j^{\prime}}^{\dagger}, \rho_{R}\right\}\right]
\end{aligned}
$$

where $\{$,$\} is the usual anticommutator. Here we have$ explicitly introduced the Hermitian and anti-Hermitian parts of the $\Gamma$ matrices, writing $\Gamma_{1 j j^{\prime}}=\gamma_{1 j j^{\prime}} / 2+i S_{1 j j^{\prime}} / \hbar$ with $\gamma_{1 j j^{\prime}}=\Gamma_{1 j j^{\prime}}+\Gamma_{1 j^{\prime} j}^{*}$ and $S_{1 j j^{\prime}}=(\hbar / 2 i)\left(\Gamma_{1 j j^{\prime}}-\Gamma_{1 j^{\prime} j}^{*}\right)$ (similar relations hold for $\Gamma_{2 j j^{\prime}}, \gamma_{2 j j^{\prime}}$, and $S_{2 j j^{\prime}}$ ). The $\gamma$ and $S$ matrices are Hermitian, i.e. $\gamma_{1,2 j j^{\prime}}^{*}=\gamma_{1,2 j^{\prime} j}$ and 
$S_{1,2 j j^{\prime}}^{*}=S_{1,2 j^{\prime} j}$. The dissipator term describes an irreversible evolution of the radiation field induced by photon emission and absorption processes. The shift $\left(H_{S}\right.$ term) corresponds to a Hamiltonian contribution to the dynamics and may be interpreted as a renormalization of the radiation energy due to the atoms present in the system. A quantity of interest is the average photon number $\left\langle N_{j}(t)\right\rangle=\operatorname{Tr}\left(\rho_{R}(t) N_{j}\right)$. It denotes the mean number of radiation quanta with the mode $j$ in the quantization volume, hence provides a measure of the radiation's total energy at time $t$. It obeys a closed evolution equation if the nondiagonal elements of the various operators in Eq. (18) are neglected:

$$
\frac{d\left\langle N_{j}\right\rangle}{d t}=\gamma_{2 j j}-\left(\gamma_{1 j j}-\gamma_{2 j j}\right)\left\langle N_{j}\right\rangle
$$

The first term of the right-hand side denotes spontaneous emission and the second term denotes absorption corrected by stimulated emission. Because of its simple interpretation, this equation has been used successfully in quantum-based deductive treatments of radiative transfer $[48,49]$.

\section{QUANTUM PHASE SPACE}

The nondiagonal matrix elements of the density operator $\rho_{R}$ in the Fock basis denote radiation coherence, a typical feature of the wave nature of light. In this section, we develop a formalism suitable to account for this property in radiative transfer. Our starting point is the master equation (18) [or, equivalently, Eqs. (5) and (6)] and its formulation within the quantum phase space formalism. The fundamental quantity of interest is the one-photon Wigner function $W(\mathbf{r}, \mathbf{p}, t)$, defined as the average of the phase space photon number operator $N(\mathbf{r}, \mathbf{p}, t)[51]$ :

$$
\begin{gathered}
W(\mathbf{r}, \mathbf{p}, t)=\operatorname{Tr}_{R}\left[\rho_{R}(t) N(\mathbf{r}, \mathbf{p})\right], \\
N(\mathbf{r}, \mathbf{p})=\frac{1}{\pi^{3} \hbar^{3}} \sum_{\boldsymbol{\varepsilon}} \int d^{3} k a_{\varepsilon}^{\dagger}\left(\frac{\mathbf{p}}{\hbar}-\mathbf{k}\right) a_{\boldsymbol{\varepsilon}}\left(\frac{\mathbf{p}}{\hbar}+\mathbf{k}\right) e^{2 i \mathbf{k} \cdot \mathbf{r}} .
\end{gathered}
$$

Here $a_{\boldsymbol{\varepsilon}}^{\dagger}(\mathbf{k})$ and $a_{\boldsymbol{\varepsilon}}(\mathbf{k})$ are the continuous versions of the creation and annihilation operators, respectively. They are related to their discrete versions as $a_{\boldsymbol{\varepsilon}_{j}}\left(\mathbf{k}_{j}\right)=$ $(L / 2 \pi)^{3 / 2} a_{j}$ and $a_{\varepsilon_{j}}^{\dagger}\left(\mathbf{k}_{j}\right)=(L / 2 \pi)^{3 / 2} a_{j}^{\dagger}$, and obey the commutation rules $\left[a_{\varepsilon}(\mathbf{k}), a_{\varepsilon^{\prime}}^{\dagger}\left(\mathbf{k}^{\prime}\right)\right]=\delta_{\varepsilon \varepsilon^{\prime}} \delta\left(\mathbf{k}-\mathbf{k}^{\prime}\right)$ and $\left[a_{\varepsilon}(\mathbf{k}), a_{\varepsilon^{\prime}}\left(\mathbf{k}^{\prime}\right)\right]=0=\left[a_{\varepsilon}^{\dagger}(\mathbf{k}), a_{\varepsilon^{\prime}}^{\dagger}\left(\mathbf{k}^{\prime}\right)\right]$. Examination of Eqs. (22) and (23) shows that $W(\mathbf{r}, \mathbf{p}, t)$ is normalized to the total number of photons. It is convenient to write the Wigner function in terms of the discretized modes

$$
\begin{aligned}
W(\mathbf{r}, \mathbf{p}, t)=\left(\frac{2}{\hbar L}\right)^{3} \sum_{j j^{\prime}} \delta_{\varepsilon_{j} \varepsilon_{j^{\prime}}} \delta & \left(\mathbf{k}_{j}+\mathbf{k}_{j}^{\prime}-\frac{2 \mathbf{p}}{\hbar}\right) \\
& \times N_{j j^{\prime}}(t) e^{-i \mathbf{k}_{j j^{\prime}} \cdot \mathbf{r}} .
\end{aligned}
$$

Here $N_{j j^{\prime}}(t)=\operatorname{Tr}\left(\rho_{R}(t) a_{j}^{\dagger} a_{j^{\prime}}\right)$ is a generalization of the average photon number defined in the previous section. This quantity obeys the following evolution equation

$\frac{d N_{j j^{\prime}}}{d t}=i \omega_{j j^{\prime}} N_{j j^{\prime}}+\gamma_{2 j j^{\prime}}-\sum_{j^{\prime \prime}}\left(\Gamma_{j j^{\prime \prime}} N_{j^{\prime \prime} j^{\prime}}+\Gamma_{j^{\prime} j^{\prime \prime}}^{*} N_{j j^{\prime \prime}}\right)$,

which is obtained from Eq. (18), using the commutation relations satisfied by the creation and annihilation operators. The first term in the right-hand side describes free evolution, i.e., in the absence of emission and absorption processes. By convention, $\omega_{j j^{\prime}}=\omega_{j}-\omega_{j^{\prime}}$. The second term is a source independent of $N_{j j^{\prime}}$ and denotes spontaneous emission. The third term denotes absorption corrected by stimulated emission. By convention, $\Gamma_{j j^{\prime}}=$ $\Gamma_{1 j j^{\prime}}^{*}-\Gamma_{2 j j^{\prime}}=\left(c \delta_{\varepsilon_{j} \varepsilon_{j^{\prime}}} / 2 L^{3}\right) \int d^{3} r e^{i \mathbf{k}_{j j^{\prime}} \cdot \mathbf{r}} \chi_{c}\left(\mathbf{r}, \hbar \mathbf{k}_{j^{\prime}}\right) . \quad \mathrm{A}$ transport equation for the Wigner function is obtained from Eqs. (24), (25), using the following relation [43]

$N_{j j^{\prime}}(t)=\left(\frac{2 \pi \hbar}{L}\right)^{3} \frac{\delta_{\boldsymbol{\varepsilon}_{j} \boldsymbol{\varepsilon}_{j^{\prime}}}}{2} \int d^{3} r e^{i \mathbf{k}_{j j^{\prime}} \cdot \mathbf{r}} W\left(\mathbf{r}, \frac{\hbar \mathbf{k}_{j}}{2}+\frac{\hbar \mathbf{k}_{j^{\prime}}}{2}, t\right)$

and taking the continuum limit:

$$
\frac{\partial W}{\partial t}(\mathbf{r}, \mathbf{p}, t)+\mathcal{T}[W](\mathbf{r}, \mathbf{p}, t)=S(\mathbf{r}, \mathbf{p}, t)-\mathcal{L}[W](\mathbf{r}, \mathbf{p}, t) .
$$

Here $\mathcal{T}$ is a functional that describes free propagation

$$
\begin{aligned}
& \mathcal{T}[W](\mathbf{r}, \mathbf{p}, t)=-\frac{c}{\pi^{2}} \mathrm{P} \int d^{3} r^{\prime} \frac{\sin \left[\frac{2 \mathbf{p}}{\hbar} \cdot\left(\mathbf{r}-\mathbf{r}^{\prime}\right)\right]}{\left|\mathbf{r}-\mathbf{r}^{\prime}\right|^{4}} \\
& \times W\left(\mathbf{r}^{\prime}, \mathbf{p}, t\right) \text {, }
\end{aligned}
$$

$S$ denotes spontaneous emission

$$
\begin{array}{r}
S(\mathbf{r}, \mathbf{p}, t)=\frac{1}{\pi^{3} \hbar^{4} p^{3}} \operatorname{Re} \int d^{3} r^{\prime} \int d^{3} p^{\prime} \eta_{c}\left(\mathbf{r}^{\prime}, \mathbf{p}^{\prime}, t\right) \\
\times e^{-2 i\left(\mathbf{r}-\mathbf{r}^{\prime}\right) \cdot\left(\mathbf{p}-\mathbf{p}^{\prime}\right) / \hbar},
\end{array}
$$

with $\eta_{c}$ being the complex emissivity

$$
\eta_{c}(\mathbf{r}, \mathbf{p}, t)=\frac{\hbar \omega_{0}}{4 \pi} A_{e g} N_{e}(\mathbf{r}, t) \phi_{c}(\omega, \hat{\mathbf{n}}, \mathbf{r}, t),
$$

( $A_{\text {eg }}$ is the corresponding Einstein coefficient) and $\mathcal{L}$ denotes the absorption corrected by stimulated emission

$$
\mathcal{L}[W](\mathbf{r}, \mathbf{p}, t)=\int d^{3} r^{\prime} \int d^{3} p^{\prime} K\left(\mathbf{r}, \mathbf{p}, t ; \mathbf{r}^{\prime}, \mathbf{p}^{\prime}\right) W\left(\mathbf{r}^{\prime}, \mathbf{p}^{\prime}, t\right),
$$

$$
\begin{aligned}
K\left(\mathbf{r}, \mathbf{p}, t ; \mathbf{r}^{\prime}, \mathbf{p}^{\prime}\right) & =\frac{c}{(\pi \hbar)^{6}} \operatorname{Re} \int d^{3} r^{\prime \prime} \int d^{3} p^{\prime \prime} \chi_{c}\left(\mathbf{r}^{\prime \prime}, \mathbf{p}^{\prime \prime}, t\right) \\
& \times e^{2 i\left[\left(\mathbf{r}-\mathbf{r}^{\prime}\right) \cdot\left(\mathbf{p}^{\prime}-\mathbf{p}^{\prime \prime}\right)-\left(\mathbf{r}-\mathbf{r}^{\prime \prime}\right) \cdot\left(\mathbf{p}-\mathbf{p}^{\prime}\right)\right] / \hbar} \cdot(32)
\end{aligned}
$$

In Eq. (28), P denotes the Cauchy principal value. Equation (27) is a generalization of the radiative transfer equation, which takes into account the wave nature of light. We refer to it as a "quantum radiative transfer equation" 
(QRTE) in the following. The space integral involved in the free propagation term $\mathcal{T}$ denotes an ambiguity in the definition of a photon trajectory if the Wigner function is not homogeneous at the radiation wavelength scale. This ambiguity is inherent to the inadequacy of using a particle picture for radiation with large wavelengths, and this inadequacy is well known in standard textbooks on radiative transfer (e.g. [2]). If the geometrical limit $(\lambda \rightarrow 0)$ is assumed, the propagation term comes down to simple advection in the phase space

$$
\mathcal{T}[W](\mathbf{r}, \mathbf{p}, t) \simeq c \hat{\mathbf{n}} \cdot \frac{\partial W}{\partial \mathbf{r}}(\mathbf{r}, \mathbf{p}, t)
$$

In the source and loss terms Eqs. (29), (31), and (32), the integrals imply delocalization over a space volume of typical extent $(\hbar / \Delta p)^{3}$, with $\Delta p$ denoting the typical momentum detuning of the atomic line under consideration. The characteristic length $\hbar / \Delta p$ corresponds to the photons' thermal de Broglie wavelength and may be identified as the radiation coherence length $\lambda^{2} / \Delta \lambda$. It is much larger than the radiation wavelength for atomic lines, which means that such a delocalization can be relevant even at the short wavelength limit. Only in the limiting case where both the Wigner function and the emission and absorption coefficients are homogeneous over this scale, the QRTE becomes local and identical with the standard radiative transfer equation, namely

$$
\left(\frac{1}{c} \frac{\partial}{\partial t}+\hat{\mathbf{n}} \cdot \frac{\partial}{\partial \mathbf{r}}\right) I=\eta-\chi I
$$

where $I=\hbar c p^{3} W$ is identified as the radiation specific intensity, $\eta=\operatorname{Re} \eta_{c}$ and $\chi=\operatorname{Re} \chi_{c}$ are the usual emission and extinction coefficients.

The QRTE can be reformulated in terms of the Moyal star product [52]. For two functions $f(\mathbf{r}, \mathbf{p}), g(\mathbf{r}, \mathbf{p})$, it is defined with the following integral

$$
\begin{aligned}
(f \star g)(\mathbf{r}, \mathbf{p})= & \frac{1}{(\pi \hbar)^{6}} \int d 1 \int d 2 f\left(\mathbf{r}+\mathbf{r}_{1}, \mathbf{p}+\mathbf{p}_{1}\right) \\
& \times g\left(\mathbf{r}+\mathbf{r}_{2}, \mathbf{p}+\mathbf{p}_{2}\right) e^{2 i\left(\mathbf{r}_{1} \cdot \mathbf{p}_{2}-\mathbf{r}_{2} \cdot \mathbf{p}_{1}\right) / \hbar}
\end{aligned}
$$

where $d 1=d^{3} r_{1} d^{3} p_{1}$ and $d 2=d^{3} r_{2} d^{3} p_{2}$ are shortcut notations for the phase space infinitesimal volume. The star product is noncommutative and has some specific properties. It can be expanded as a power series of $\hbar$ and reduces to simple multiplication in the limit $\hbar \rightarrow 0$. An application of the series expansion will be examined in Sec. VI. In terms of the star product, the propagation term takes a simple form

$$
\mathcal{T}[W]=c\{W,|\mathbf{p}|\}_{M},
$$

where $\{f, g\}_{M}=(f \star g-g \star f) / i \hbar$ is the so-called Moyal bracket. In the limit $\hbar \rightarrow 0$, it is equivalent to the Poisson bracket $\{f, g\}=(\partial f / \partial \mathbf{r}) \cdot(\partial g / \partial \mathbf{p})-(\partial f / \partial \mathbf{p}) \cdot(\partial g / \partial \mathbf{r})$ and the propagation term reduces to advection, Eq. (33). The source and loss terms can be written as follows

$$
S(\mathbf{r}, \mathbf{p}, t)=\frac{1}{\hbar p^{3}} \lim _{\substack{\mathbf{r}_{0} \rightarrow \mathbf{r} \\ \mathbf{p}_{0} \rightarrow \mathbf{p}}} \operatorname{Re}\left(\eta_{c} \star \Delta_{\mathbf{r}_{0}, \mathbf{p}_{0}}^{*}\right)(\mathbf{r}, \mathbf{p}, t),
$$

$$
\mathcal{L}[W](\mathbf{r}, \mathbf{p}, t)=c \lim _{\substack{\mathbf{r}_{0} \rightarrow \mathbf{r} \\ \mathbf{p}_{0} \rightarrow \mathbf{p}}} \operatorname{Re}\left(\left(W \Delta_{\mathbf{r}_{0}, \mathbf{p}_{0}}^{*}\right) \star \chi_{c}\right)(\mathbf{r}, \mathbf{p}, t),
$$

where $\Delta_{\mathbf{r}_{0}, \mathbf{p}_{0}}(\mathbf{r}, \mathbf{p})=\exp \left[2 i\left(\mathbf{r}-\mathbf{r}_{0}\right) \cdot\left(\mathbf{p}-\mathbf{p}_{0}\right) / \hbar\right] \equiv$

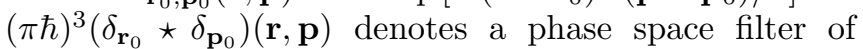
characteristic volume $\hbar^{3}$ [with $\delta_{\mathbf{r}_{0}}(\mathbf{r})=\delta\left(\mathbf{r}-\mathbf{r}_{0}\right)$ and $\delta_{\mathbf{p}_{0}}(\mathbf{p})=\delta\left(\mathbf{p}-\mathbf{p}_{0}\right)$ being the usual delta functions in the $\mathbf{r}-$ and $\mathbf{p}$-spaces]. They reduce to $\eta / \hbar p^{3}$ and $c \chi W$ in the limit $\hbar \rightarrow 0$ and the QRTE is equivalent to the standard radiative transfer equation (34).

\section{MODELING OF DETECTOR FUNCTIONS}

The quantum radiative transfer equation (27) has not necessarily a positive-definite solution, even though the initial and/or bondary conditions are positive, so that a probabilistic interpretation is not straightforward. This issue stems from the Heisenberg uncertainty principle. It is customary to introduce a coarse-graining with a phase space cell of size larger than $\hbar^{3}$. This procedure amounts to considering the following quantity

$$
\bar{W}(\mathbf{r}, \mathbf{p}, t)=\int d^{3} r^{\prime} \int d^{3} p^{\prime} f\left(\mathbf{r}, \mathbf{p} ; \mathbf{r}^{\prime}, \mathbf{p}^{\prime}, t\right) W\left(\mathbf{r}^{\prime}, \mathbf{p}^{\prime}, t\right),
$$

instead of the Wigner function, and to interpret it as a probability density function. The explicit expression for the smoothing function $f\left(\mathbf{r}, \mathbf{p} ; \mathbf{r}^{\prime}, \mathbf{p}^{\prime}, t\right)$ depends on the problem under consideration. For example, a Gaussian function $f\left(\mathbf{r}, \mathbf{p} ; \mathbf{r}^{\prime}, \mathbf{p}^{\prime}, t\right)=\exp \left[-a\left(\mathbf{r}-\mathbf{r}^{\prime}\right)^{2} / \hbar-(\mathbf{p}-\right.$ $\left.\left.\mathbf{p}^{\prime}\right)^{2} / a \hbar\right] /(\pi \hbar)^{3}$ (with $a>0$ ) leads to the so-called Husimi distribution, positive everywhere (e.g. [53]). Other smoothing functions have been proposed (even for the radiative transfer equation, e.g. [31, 32]). There is a correspondence between Eq. (39) and "observable" quantities in the terminology of Monte-Carlo kinetic simulations. The phase space integral denotes a particular case of a "response" function, namely the response of a detector characterized by the $f$ function to the radiation field (e.g. [54, 55]). For photons, as well as for massive particles, the quantities involved in fluid equations (density, temperature, etc.) can be written as such a phase space integral, and, hence, are other particular cases of response functions. The point of view of using response functions is practical if one needs the description of a photodetection signal, e.g., from a spectrometer. To illustrate it we consider as a particular case an isolated atom in its ground state $|g\rangle$, located at $\mathbf{r}=\mathbf{0}$. A photon is considered detected if the atom is ionized after interaction with the radiation field. Denoting $|a\rangle$ an ionization state and $P_{g \rightarrow a}$ the probability of transition $g \rightarrow a$, we define the photo-detection signal at time $t$ as [47]

$$
P_{\text {ion }}(t)=\sum_{a} R_{a} P_{g \rightarrow a}(t)
$$

where $R_{a}$ is the efficiency of detection after ionization in the $|a\rangle$ state. The probability $P_{g \rightarrow a}$ can be evaluated 
using the standard perturbation theory. As in Sec. II, dipole interaction may be considered. If we neglect nonresonant terms, we get, at second order in $V$

$$
P_{g \rightarrow a}(t)=\frac{\left|\mathbf{d}_{a g}\right|^{2}}{3 \hbar^{2}} \int_{0}^{t} d t^{\prime} \int_{0}^{t} d t^{\prime \prime} G\left(t^{\prime \prime}, t^{\prime}\right) e^{i \omega_{a g}\left(t^{\prime}-t^{\prime \prime}\right)}
$$

where $G\left(t^{\prime}, t^{\prime \prime}\right)=\left\langle\mathbf{E}^{(-)}\left(t^{\prime \prime}\right) \cdot \mathbf{E}^{(+)}\left(t^{\prime}\right)\right\rangle$ denotes a correlation function of the electric field, with $\mathbf{E}^{(+)}=$ $\sum_{j} i\left(\hbar \omega_{j} / 2 \varepsilon_{0} L^{3}\right)^{1 / 2} \varepsilon_{j} a_{j}$ and $\mathbf{E}^{(-)}=\mathbf{E}^{(+) *}$. The transition probability can be written as a Fourier transform

$P_{\text {ion }}(t)=\frac{1}{2 \pi} \int_{0}^{t} d t^{\prime} \int_{0}^{t} d t^{\prime \prime} \int_{-\infty}^{+\infty} d \omega s(\omega) e^{i \omega\left(t^{\prime}-t^{\prime \prime}\right)} G\left(t^{\prime \prime}, t^{\prime}\right)$,

where $s(\omega)$ is given by

$$
s(\omega)=\frac{2 \pi}{3 \hbar^{2}} \sum_{a} R_{a}\left|\mathbf{d}_{a g}\right|^{2} \delta\left(\omega-\omega_{a g}\right) .
$$

and denotes the spectral sensitivity of the detector. A correspondence with the Wigner function is obtained from Eq. (26), identifying the correlation function as a phase space average at initial time

$$
\begin{array}{r}
G\left(t^{\prime \prime}, t^{\prime}\right)=\frac{\hbar \omega_{0}}{2 \varepsilon_{0}(\pi \hbar)^{3}} \int d^{3} r \int d^{3} p \int d^{3} p^{\prime} W(\mathbf{r}, \mathbf{p}, 0) \\
\times e^{2 i \mathbf{p}^{\prime} \cdot \mathbf{r} / \hbar} e^{i c\left[\left|\mathbf{p}+\mathbf{p}^{\prime}\right| t^{\prime \prime}-\left|\mathbf{p}-\mathbf{p}^{\prime}\right| t^{\prime}\right] / \hbar} .
\end{array}
$$

Here, for simplicity it has been assumed that the Wigner function evolves at a time scale much larger than the radiation's characteristic period $\left(\sim \omega_{0}^{-1}\right)$ and coherence time $\left(\sim \Delta \omega_{0}^{-1}\right.$, with $\Delta \omega_{0}$ being the characteristic spectral band). If the radiation is homogeneous at the detector's location, one can set $W(\mathbf{r}, \mathbf{p}, 0) \simeq W(\mathbf{0}, \mathbf{p}, 0) \equiv W(\mathbf{p})$ in Eq. (44). The correlation function depends on the difference $t^{\prime}-t^{\prime \prime}$ only

$$
G\left(t^{\prime \prime}, t^{\prime}\right)=\frac{\hbar \omega_{0}}{2 \varepsilon_{0}} \int d^{3} p W(\mathbf{p}) e^{i c p\left(t^{\prime \prime}-t^{\prime}\right) / \hbar} \equiv G\left(t^{\prime}-t^{\prime \prime}\right),
$$

and a simple expression holds for the transition probability at large times

$$
P_{\text {ion }}(t) \sim \Gamma t
$$

where

$$
\Gamma=\frac{\hbar \omega_{0}}{2 \varepsilon_{0}} \int d^{3} p W(\mathbf{p}) s\left(\frac{c p}{\hbar}\right)
$$

This quantity can be interpreted as a transition rate and it provides a response function. Equation (47) is a phase space adaptation of the formula involved in the modeling of photodetection signals. The photon is detected if its energy $p c$ is in the typical spectral band of the $s$ function. For small-band detector, the transition rate is proportional to the Wigner function and the latter can be interpreted as a Fourier transform of the electric field's autocorrelation function. On the contrary, a large band detector yields a signal proportional to the Wigner function integrated over the momentum, and the latter is proportional to the square of the electric field amplitude. The method described above can be adapted to realistic detectors used in plasma spectroscopy. A generalization of Eq. (47), involving integral over the spatial extent of the detector, can be obtained in a straightforward manner, taking the transition probability $P_{\text {ion }}$ for a set of atoms instead of one atom only. Such a treatment requires the detector's geometry be well accounted for, and, hence, is strongly dependent of the experimental device.

\section{HOLSTEIN-BIBERMAN THEORY}

The Holstein-Biberman theory [56, 57] provides a description of opacity effects based on the modeling of atomic excited levels, with no explicit reference to the radiative transfer equation. Take a set of two-level atoms and, for simplicity, neglect their interaction with massive particles (collisions). The following evolution equation is obtained ("Holstein-Biberman equation")

$$
\frac{\partial N_{2}}{\partial t}(\mathbf{r}, t)=-\gamma N_{2}(\mathbf{r}, t)+\gamma \int d^{3} r^{\prime} G\left(\mathbf{r}, \mathbf{r}^{\prime}\right) N_{2}\left(\mathbf{r}^{\prime}, t\right) .
$$

It provides a closed relation for the density of excited atoms, $N_{2}$. The rate $\gamma$ denotes the reciprocal of the life time for the excited state (here $\gamma \equiv A_{21}$ since collisions are neglected), and $G\left(\mathbf{r}, \mathbf{r}^{\prime}\right)$ is a kernel that describes the production of excited atoms at $\mathbf{r}$ given deexcitation at $\mathbf{r}^{\prime}$. An explicit expression is obtained from the solution of the standard radiative transfer equation (34) in the adiabatic limit $(\partial / \partial t \equiv 0)$, using the identity

$$
\begin{aligned}
& \gamma \int d^{3} r^{\prime} G\left(\mathbf{r}, \mathbf{r}^{\prime}\right) N_{2}\left(\mathbf{r}^{\prime}, t\right) \\
& =N_{1} B_{12} \int d \omega \int \frac{d \Omega}{4 \pi} \phi(\omega, \hat{\mathbf{n}}) I(\omega, \hat{\mathbf{n}}, \mathbf{r}, t),
\end{aligned}
$$

where $B_{12}$ is the Einstein coefficient for absorption (stimulated emission is neglected for simplicity) and $N_{1}$ is the density of atoms in the fundamental state. The latter, as well as the line shape function $\phi(\omega, \hat{\mathbf{n}})$, are assumed here space and time independent. For simplicity we also assume isotropic line shape function, i.e. $\phi(\omega, \hat{\mathbf{n}}) \equiv \phi(\omega)$ and $\chi(\mathbf{p}) \equiv \chi(\omega)$. The solution of the radiative transfer equation [2] leads to an expression for $G$ that depends on $\left|\mathbf{r}-\mathbf{r}^{\prime}\right|$ only, namely

$$
G\left(\mathbf{r}, \mathbf{r}^{\prime}\right) \equiv G(s)=-\frac{1}{4 \pi s^{2}} \frac{d T}{d s}(s)
$$

where $s=\left|\mathbf{r}-\mathbf{r}^{\prime}\right| \equiv|\mathbf{s}|$ and $T(s)$ is referred to as the "transmission factor". It denotes the probability that an emitted photon travels the distance $s$ without being absorbed, it is defined by

$$
T(s)=\int d \omega \phi(\omega) e^{-\chi(\omega) s},
$$


and it satisfies $T(0)=1$ and $T(s) \rightarrow 0$ when $s \rightarrow \infty$. A related quantity is the escape factor (with some different conventions in the definition [58]) used in collisionalradiative codes (e.g. $[10,11,59]$ ).

The treatment above can be adapted in such a way to account for spatial coherence. A preliminary investigation [44] has indicated the possibility for an alteration of escape factors if the coherence length is of the same order as the photon mean free path and/or the medium's characteristic size. In that work, the model was based on the quantum transport equation (27) and the correspondence $I \leftrightarrow \hbar c p^{3} W$ was used in Eq. (49). This is not fully consistent if coherence effects are present, because the integral in the right-hand side of Eq. (49) implies local interaction. A more precise development can be done along the lines of the formalism developed in Secs. II and III, writing down a master equation for the atomic populations from first principles. It is obtained from an equation for the atomic density matrix $\rho_{A}=\operatorname{Tr}_{R}(\rho)$ formally identical to Eq. (5). We do not give details on the procedure because it is similar to that done for photons in Sec. II. The resulting evolution equation for the population of the excited level reads

$$
\frac{\partial N_{2}}{\partial t}(\mathbf{r}, t)=-A_{21} N_{2}(\mathbf{r}, t)+\mathcal{B}[W](\mathbf{r}, t),
$$

where the functional $\mathcal{B}$ denotes absorption and provides a source of excited atoms. It is nonlocal in phase space, in the same way as the quantum radiative transfer equation (27), and it is related to the loss term $\mathcal{L}[W]$ [Eqs. (31) and (32), or Eq. (38)] through the following integral

$$
\mathcal{B}[W](\mathbf{r}, t)=\int d^{3} p \mathcal{L}[W](\mathbf{r}, \mathbf{p}, t) .
$$

An analysis of the quantum radiative transfer equation indicates that the photon density (first-order moment, $\left.\mathrm{m}^{-3}\right) N(\mathbf{r}, t)=\int d^{3} p W(\mathbf{r}, \mathbf{p}, t)$ obeys the same evolution equation as does the excited level population Eq. (52), up to a minus sign in the source and loss terms, which indicates a conservation of the total (atoms + photons) particle density. A closed equation for the atomic population is obtained by inserting the solution of the quantum radiative transfer equation in $\mathcal{B}[W]$. Assuming homogeneous and stationary absorption coefficient $[\chi(\mathbf{r}, \mathbf{p}, t) \equiv \chi(\mathbf{p})]$, and taking the adiabatic limit $(\partial / \partial t \equiv 0)$, the QRTE reads

$$
c \hat{\mathbf{n}} \cdot \frac{\partial W}{\partial \mathbf{r}}(\mathbf{r}, \mathbf{p}, t)=S(\mathbf{r}, \mathbf{p}, t)-\mathcal{L}[W](\mathbf{r}, \mathbf{p}, t),
$$

where the loss term involves a space integral only, of convolution-type

$$
\begin{aligned}
& \mathcal{L}[W](\mathbf{r}, \mathbf{p}, t)=\int d^{3} r^{\prime} K_{0}\left(\mathbf{r}-\mathbf{r}^{\prime}, \mathbf{p}\right) W\left(\mathbf{r}^{\prime}, \mathbf{p}, t\right), \\
& K_{0}(\mathbf{r}, \mathbf{p})=\frac{c}{(\pi \hbar)^{3}} \operatorname{Re} \int d^{3} p^{\prime} \chi_{c}\left(\mathbf{p}^{\prime}\right) e^{2 i \mathbf{r} \cdot\left(\mathbf{p}-\mathbf{p}^{\prime}\right) / \hbar} .
\end{aligned}
$$

The solution has a simple structure in the Fourier space

$$
\hat{W}(\mathbf{k}, \mathbf{p}, t)=\frac{\hat{S}(\mathbf{k}, \mathbf{p}, t)}{i c \hat{\mathbf{n}} \cdot \mathbf{k}+\hat{K}_{0}(\mathbf{k}, \mathbf{p})} .
$$

Here, by convention, $\hat{f}(\mathbf{k})=\int d^{3} r f(\mathbf{r}) \exp (-i \mathbf{k} \cdot \mathbf{r})$ for any function of the space coordinates $f(\mathbf{r})$. A generalization of the Holstein-Biberman kernel $G\left(\mathbf{r}, \mathbf{r}^{\prime}\right)$ is thus obtained from Eq. (52), using the identity

$$
\gamma \int d^{3} r^{\prime} G\left(\mathbf{r}, \mathbf{r}^{\prime}\right) N_{2}\left(\mathbf{r}^{\prime}, t\right)=\mathcal{B}[W](\mathbf{r}, t)
$$

taking the solution (57), and using that $\hat{S}$ is proportional to the Fourier transform of the population $N_{2}$. The calculation, tedious but with no major difficulties, yields an isotropic kernel $\left[G\left(\mathbf{r}, \mathbf{r}^{\prime}\right) \equiv G(s)\right]$ with a rather simple expression in the Fourier space

$$
\hat{G}(\mathbf{k})=\int d \omega \int \frac{d \Omega}{4 \pi} \frac{\psi(\mathbf{p}, \mathbf{k}) \hat{K}_{0}(\mathbf{k}, \mathbf{p})}{i c \hat{\mathbf{n}} \cdot \mathbf{k}+\hat{K}_{0}(\mathbf{k}, \mathbf{p})} .
$$

Here, by definition, $\hat{G}(\mathbf{k})=\int d^{3} s G(s) \exp (-i \mathbf{k} \cdot \mathbf{s})$ and $\psi(\mathbf{p}, \mathbf{k})=\left[\phi_{c}(\mathbf{p}+\hbar \mathbf{k} / 2)+\phi_{c}^{*}(\mathbf{p}-\hbar \mathbf{k} / 2)\right] / 2$ [with the convention $\left.\phi_{c}(\omega=|\mathbf{p}| c / \hbar, \hat{n}=\mathbf{p} /|\mathbf{p}|) \equiv \phi_{c}(\mathbf{p})\right]$. A similar expression has been obtained in [60] in a different context, but there is no clear interpretation of the role of coherence.

A generalization of the transmission factor, accounting for coherence effects, can be obtained from Eq. (50), taking the inverse Fourier transform of Eq. (59). Its probabilistic interpretation is not straightforward because this quantity can be negative in the same way as the Wigner function. We have estimated the transmission factor in the case of a Doppler-broadened spectral line, taking the Maxwellian velocity distribution for the atoms. The complex line shape depends on the frequency detuning $\Delta \omega=\omega-\omega_{0}$ only and is given by

$$
\phi_{c}(\Delta \omega)=\frac{1}{\Delta \omega_{D} \sqrt{\pi}} w^{*}\left(\frac{\Delta \omega}{\Delta \omega_{D}}\right),
$$

where $\Delta \omega_{D}=\omega_{0} v_{0} / c$ is the Doppler width, with $v_{0}=$ $(2 T / m)^{1 / 2}$ being the atoms' thermal velocity at temperature $T$, and $w(z)$ is the complex Faddeeva function [61]. The real part of $\phi_{c}$ is the usual Gaussian shape; the imaginary part is an odd, slowly decreasing function of $|\Delta \omega|$ (see Fig. 1). To simplify the numerical calculation we have used the approximation $|\mathbf{p} \pm \hbar \mathbf{k} / 2| \simeq|\mathbf{p}| \pm \hbar \mathbf{k} \cdot \hat{\mathbf{n}} / 2$ in the evaluation of $\psi(\mathbf{p}, \mathbf{k})$ which stems from the orderings $|\Delta \omega|, k c \ll \omega_{0}$ ( $k$ is estimated as the inverse gradient length of the Wigner function). A further simplification is provided by the substitution $\phi_{c}(\Delta \omega \pm \hat{\mathbf{n}} \cdot \mathbf{k} c / 2) \leftrightarrow \phi_{c}\left(\Delta \omega \pm i \chi_{0} c / 2\right)$ where $\chi_{0}=\chi(\Delta \omega=0)$ is the real absorption coefficient $\left(\mathrm{m}^{-1}\right)$ at the line center. This substitution is suggested from the structure of the integral in Eq. (59). The presence of a denominator indicates that the integrand is significant when $\left|i c \hat{\mathbf{n}} \cdot \mathbf{k}+\hat{K}_{0}(\mathbf{k}, \mathbf{p})\right|$ is small. If one allows $\mathbf{k}$ to have 


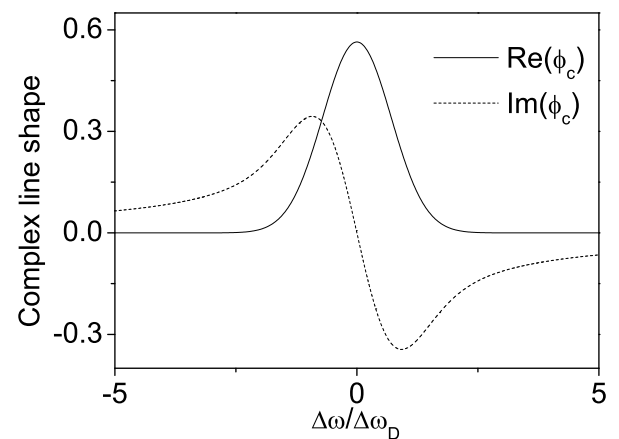

FIG. 1: Plot of the complex Doppler line shape. The real part is the usual Gaussian shape and the imaginary part is an odd, slowly decreasing function of $|\Delta \omega|$.

complex coordinates, a sufficient condition is provided by the relation $i c \hat{\mathbf{n}} \cdot \mathbf{k} \simeq-\hat{K}_{0}(\mathbf{k}, \mathbf{p})$. Evaluating the latter term by its real part at the line center, $-\chi_{0}$, yields the approximation. It should be noted that this substitution is drastic, because the possibility for allowing $\mathbf{k}$ to be a complex vector is not guaranteed. Here our motivation is given by the need of an analytical evaluation of the inverse Fourier transform of $\hat{G}(\mathbf{k})$, hence, we leave detailed mathematical analysis as a further work. Since now $\phi_{c}$ is independent of the Fourier variable, this also holds for $\psi$ and $K_{0}$ and one can write $\psi(\mathbf{p}, \mathbf{k}) \equiv \psi(\Delta \omega)$ and $\hat{K}_{0}(\mathbf{k}, \mathbf{p}) \equiv K_{0}(\Delta \omega)$; the inverse Fourier transform is analytical and the corresponding transmission factor has a simple form

$$
T(s)=\operatorname{Re} \int d \Delta \omega \psi(\Delta \omega) e^{-K_{0}^{*}(\Delta \omega) s},
$$

which is similar to that obtained within the usual radiative transfer theory, Eq. (51). Figure 2 shows a plot of the transmission factor for finite coherence length $\lambda_{c}$, taking $\lambda_{c}=c / \Delta \omega_{D}$ as a definition and assuming various values for the adimensional parameter $\chi_{0} \lambda_{c}$. The latter characterizes the amplitude of the modifications in the radiation field induced by spatial coherence. As can be seen, the presence of spatial coherence alters the transmission factor, with a strong deviation at large penetration length. It is not clear that such deviations are directly observable given the non-positivity of this quantity and the large number of approximations used in our treatment, but this result suggests that an accurate modeling of radiative transfer in optically thick medium should involve a more advanced formalism than the usual one.

\section{CALCULATIONS OF ABSORPTION LINES}

The QRTE provides a tempting approach for retaining coherence in radiative transfer models but practical

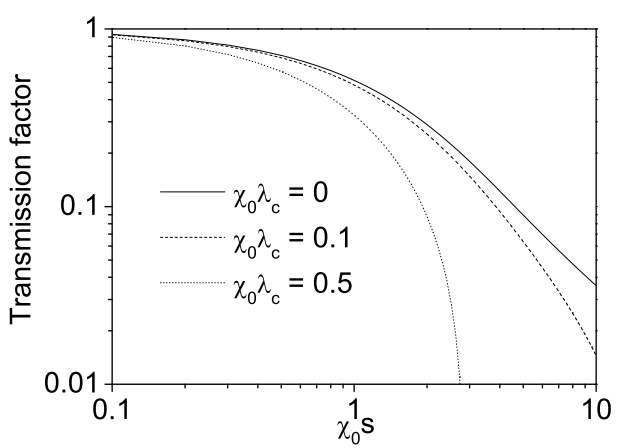

FIG. 2: Generalized transmission factor for Doppler broadening, according to Eq. (61). Strong deviations are present at large penetration length if the coherence length $\lambda_{c}$ is significant.

applications in realistic cases are tricky, given the nonlocality of the equation and the oscillating behavior of the various integrands. By analogy with Wigner transport equations for massive particles, it is of interest to consider series expansion of the star product in powers of $\hbar$. We give in this section an overview of such a procedure for a specific problem, the absorption of a large band radiation by an atomic mixture. Such a problem arises typically in high-density plasma physics, where specific experimental campaigns have been carried out [3]. We focus on the irradiation of a pure Magnesium plasma in a slab of size $L$ at $z=0$ and we examine the absorption line $\mathrm{Mg}^{11+} 1 \mathrm{~s}_{1 / 2}-2 \mathrm{p}_{3 / 2}$ (Ly- $\alpha$, blue component). Consider the first-order approximation of the star product

$$
f \star g \simeq f g+\frac{i \hbar}{2}\{f, g\},
$$

where $f$ and $g$ are two arbitrary phase space functions. The quantum radiative transfer equation within this approximation has the general form

$$
\begin{array}{r}
{\left[\frac{\partial}{\partial t}+c\left(\hat{\mathbf{n}}-\frac{\hbar}{2} \frac{\partial \chi_{I}}{\partial \mathbf{p}}\right) \cdot \frac{\partial}{\partial \mathbf{r}}+\frac{c \hbar}{2} \frac{\partial \chi_{I}}{\partial \mathbf{r}} \cdot \frac{\partial}{\partial \mathbf{p}}\right] W} \\
=\frac{\eta}{\hbar p_{0}^{3}}-c \chi W
\end{array}
$$

and reduces to

$$
\left(\hat{n}_{z}-\frac{\hbar}{2} \frac{\partial \chi_{I}}{\partial p_{z}}\right) \frac{\partial W}{\partial z}=-\chi W
$$

if stationarity and homogeneity are assumed, and selfemission is neglected. In Eqs. (63) and (64), $\chi_{I}$ denotes the imaginary part of the complex absorption coefficient. The Wigner function is space-dependent through the $z$ coordinate only and can be expressed as an exponential

$$
W(z, \mathbf{p})=W_{0} \exp -\left[\frac{\chi(\mathbf{p}) z}{\hat{n}_{z}-\frac{\hbar}{2} \frac{\partial \chi_{I}}{\partial p_{z}}(\mathbf{p})}\right] .
$$


Here $W_{0}$ denotes the value taken by the Wigner function at the boundary $z=0$. It does not depend on the momentum $\mathbf{p}$ because large band radiation is considered. We have compared this model to the solution of the QRTE for radiation propagating to the left $(\mathbf{p} \equiv|\mathbf{p}| \hat{z})$. The approximation $W\left(\mathbf{r}^{\prime}, \mathbf{p}^{\prime}\right) \simeq W\left(\mathbf{r}^{\prime}, \mathbf{p}\right)$ has been used in Eq. (27) and the boundary condition $W(z<0, \mathbf{p})=0$, $W(z=0, \mathbf{p})=1$ has been assumed (note, the setting of boundary conditions on a Wigner function is not straightforward, e.g. [62, 63]). The QRTE has been rewritten in integral form, setting $W(z, \mathbf{p}) \equiv W(z, \Delta \omega)$,

$$
W(z, \Delta \omega)=H(z)-\int_{0}^{\infty} d z^{\prime} K_{1}\left(z, z^{\prime}, \Delta \omega\right) W\left(z^{\prime}, \Delta \omega\right),
$$

and it has been solved numerically. For simple evaluation of the kernel $K_{1}$ we have assumed Doppler and natural broadening only (a more elaborate line shape model should include Stark broadening, e.g. [64, 65] for recent works). The complex line shape function is analytical and the corresponding kernel reads

$$
\begin{array}{r}
K_{1}\left(z, z^{\prime}, \Delta \omega\right)=\chi_{0} \operatorname{Re}\left\{e ^ { - \Omega ^ { 2 } } \left[-H\left(m-z^{\prime}\right) \operatorname{erfc}(i \Omega)\right.\right. \\
-H\left(z^{\prime}-m\right) \operatorname{erfc}\left(i \Omega-\frac{z^{\prime}-m}{\lambda_{c}}\right) \\
\left.\left.+\operatorname{erfc}\left(i \Omega-\frac{z^{\prime}}{\lambda_{c}}\right)\right]\right\}
\end{array}
$$

where $H$ is the Heaviside step function, erfc is the complementary error function, $\Omega=\Delta \omega / \Delta \omega_{D}+i A_{2 p 1 s} / 2 \Delta \omega_{D}$ and $m=\min (z, L)$ are shortcut notations, and $\lambda_{c}=$ $c / \Delta \omega_{D}$ denotes the Doppler coherence length. A specific result is shown in Figs. 3 and 4 . The Wigner function of the radiation at the line center $W(z, \Delta \omega=0)$ and the spectral profile of the outgoing radiation $W(z>L, \Delta \omega)$ are plotted. The calculations have been done for $T=160$ $\mathrm{eV}$, assuming $N=4 \times 10^{19} \mathrm{~cm}^{-3}$ for the density of absorbers $\left(\mathrm{Mg}^{11+}\right.$ in the $1 \mathrm{~s}_{1 / 2}$ state). At such conditions the coherence length is comparable to the photon mean free path, with $\chi_{0} \lambda_{c}=0.7\left(\lambda_{c}=c / \Delta \omega_{D}\right.$ is used as an estimate for the coherence length because $A_{2 p 1 s} / 2 \Delta \omega_{D}$ does not exceed $3 \%$ here). The photon mean free path is estimated as $\chi_{0}^{-1} \simeq 1.7 \times 10^{-6} \mathrm{~m}$. The size $L$ has been set equal to $10 \chi_{0}^{-1}$, in such a way that absorption effects are significant. As shown in the figures, the coherence alters the radiation penetration and yields a significant change in the absorption spectrum. The slower decrease of the Wigner function suggests that coherence leads to a reduction of the opacity. This result is in agreement with the previous investigations reported in $[44,45]$. We have compared the quantum transport model [Eqs. (66), (67)] to the first-order approximation [Eq. (65) with $W_{0} \equiv 1$ ]. The results are in a very good agreement for small values of $\chi_{0} \lambda_{c}$. An example is given in Fig. 5, where a plot of the penetration for $\chi_{0} \lambda_{c}=0.2$ is shown. The calculation was done assuming $L=10 \chi_{0}^{-1}$ again and taking $A_{2 p 1 s} / 2 \Delta \omega_{D}=0.02$. Deviations are obtained at larger coherence length, because a first-order approximation is

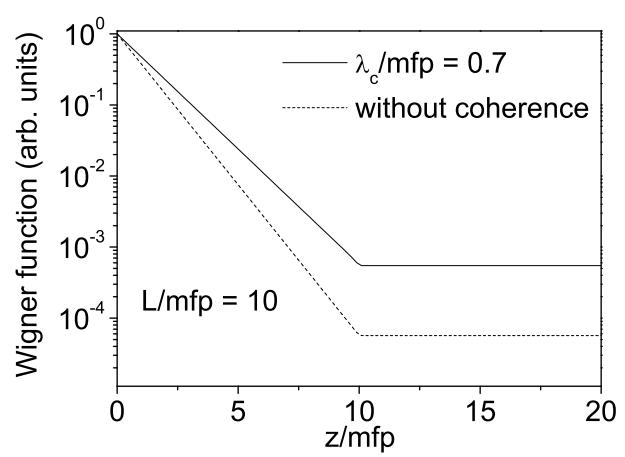

FIG. 3: Plot of the Wigner function at the line center $(\Delta \omega=0)$, for large-band incident radiation propagating in an optically thick medium. "mfp" denotes the photon mean free path, identical to $\chi_{0}^{-1}$. The attenuation is weaker when radiation coherence is retained (solid line).

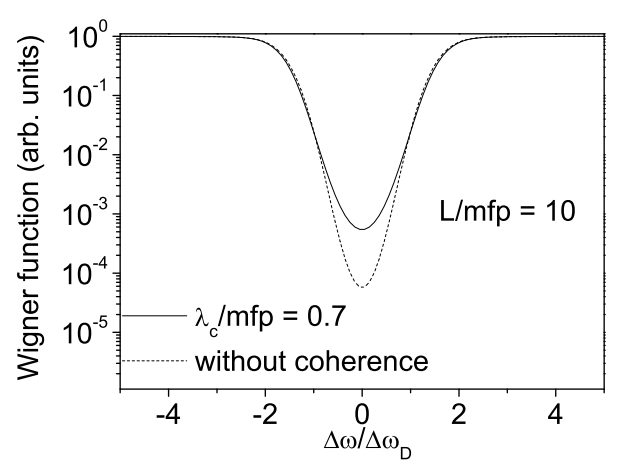

FIG. 4: Radiation spectrum outside the medium, at $z=2 L$, neglecting (dashed line) and retaining (solid line) coherence. Significant modifications are present at the line center.

inappropriate by definition in this case (Figs. 6 and 7). A more precise approximation scheme, e.g., involving expansion to second-order in $\hbar$, would provide a better result.

\section{APPLICATION TO LASER PHYSICS}

The radiative transfer theory is used for design purposes in laser physics. A typical problem is to find the optimal resonator parameters (such as the mirrors' reflectivity) which maximize the power output (e.g. [66-68]). The usual treatments are based on the standard radiative transfer equation (34) coupled to a collisional-radiative model. In the simplest case, one assumes the population of atoms in the upper laser level given by the following balance relation (e.g. [69])

$$
R_{p}=\left[\frac{1}{\tau}+B_{21} \bar{I}(z)\right] N_{2}(z)
$$




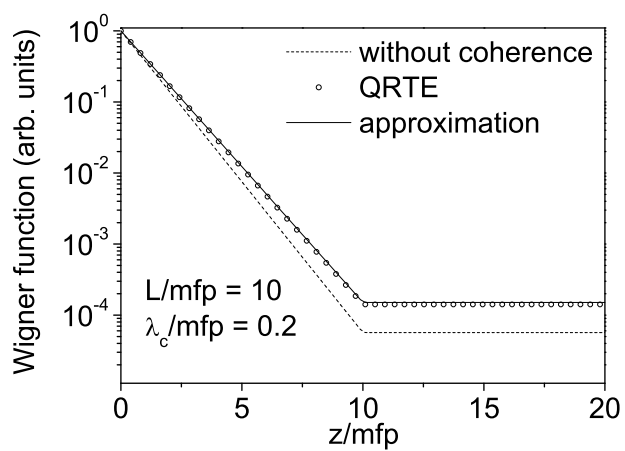

FIG. 5: Plot of the Wigner function at $\Delta \omega=0$ obtained within the first-order approximation (solid line) and solving the quantum radiative transfer equation (QRTE) in integral form, Eq. (66) (circles). Also shown in the plot is the result of the standard radiative transfer equation (dashed line). The first-order approximation provides a very good estimate of coherence effects.

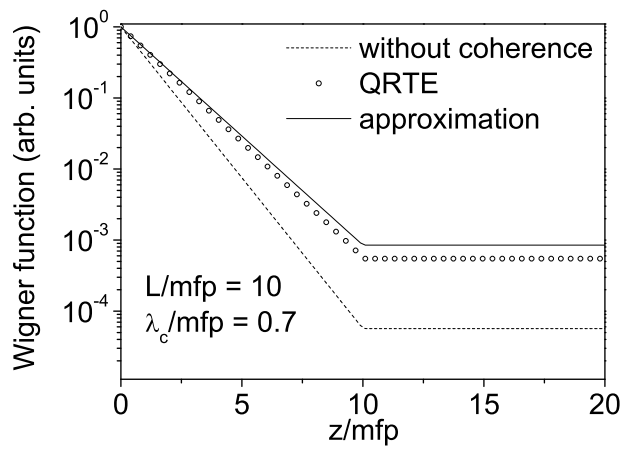

FIG. 6: The first-order approximation is restricted to small values of $\chi_{0} \lambda_{c}$. Here, plot of the Wigner function at $\Delta \omega=$ 0 for $\chi_{0} \lambda_{c}=0.7$. The approximation yields a significant deviation, with an underestimate of the radiation attenuation.

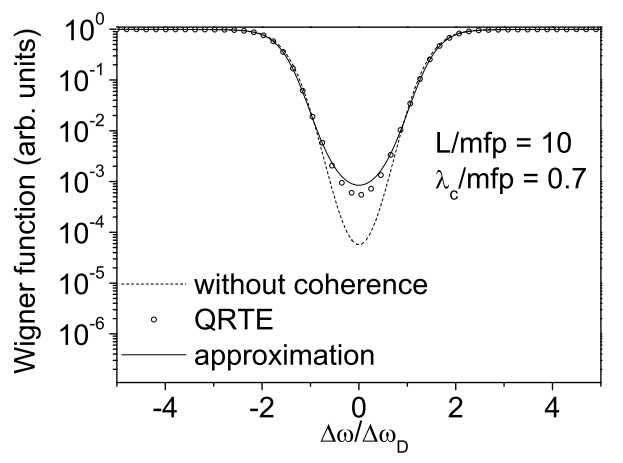

FIG. 7: Deviations are also expected on the line shape. Here plot of the radiation spectrum at $z=2 L$, with the same conditions as Fig. 6. where $R_{p}$ is the pump rate $\left(\mathrm{m}^{-3} \times \mathrm{s}^{-1}\right)$ into the upper level, $\tau$ denotes the lifetime of the level due to spontaneous emission and collisional quenching, $B_{21}$ is the Einstein coefficient of the laser transition, $\bar{I}(z)=$ $\int d \omega \int d \Omega \phi(\omega, \hat{\mathbf{n}}) I(\omega, \hat{\mathbf{n}}, z) / 4 \pi$ is the specific intensity averaged with the line shape function, and $z$ denotes the position inside the cavity $(0<z<L$, see Fig. 8$)$. The laser flux $\left(\mathrm{W} / \mathrm{m}^{2}\right)$ is the superposition of two counterpropagating components $I_{+}$and $I_{-}$. They obey the following differential equation

$$
\pm \frac{d I_{ \pm}}{d z}(z)=[g(z)-\alpha] I_{ \pm}(z)
$$

which stems from the RTE in stationary regime, assuming the spontaneous emission negligible. $g(z)$ denotes the gain at the line center, identical to the opposite of the extinction coefficient and positive for normal laser operation, and $\alpha$ stands for nonsaturable losses. Equations (68) and (69) form a closed set, which provides the specific intensity in the cavity and yields an expression for the laser output power. For example, the treatment reported in [69] for a $\mathrm{KrF}$ laser yields the following result

$$
I_{\text {out }}=t_{2} I_{S} \sqrt{\frac{\delta}{r_{2}}}
$$

where $I_{S}=4 \pi /\left(B_{21} \phi_{0} \tau\right)$ is the so-called saturation flux $\left[\phi_{0}\right.$ denotes the line shape function evaluated at the line center], $t_{2}, r_{2}$ are the transmission and reflection coefficients of the output coupler, and $\delta$ is an adimensional parameter determined from a transcendental equation

$\ln \sqrt{r_{1} r_{2}}-\alpha L=\frac{\gamma}{p_{+}-p_{-}} \times \ln \left[\frac{\left(\sqrt{\delta / r_{2}}-p_{+}\right)\left(\sqrt{\delta r_{1}}-p_{-}\right)}{\left(\sqrt{\delta / r_{2}}-p_{-}\right)\left(\sqrt{\delta r_{1}}-p_{+}\right)}\right]$,

which is obtained from algebraic manipulations involving the boundary conditions. $\gamma=g_{0} / \alpha$ and $p_{ \pm}=(1 / 2)[\gamma-$ $\left.1 \pm \sqrt{(\gamma-1)^{2}-4 \delta}\right]$ are adimensional parameters. $g_{0}=$ $\hbar \omega_{0} B_{21} \phi_{0} R_{p} \tau / 4 \pi$ is the so-called small-signal gain.

We have adapted this model in such a way to account for coherence effects. The simplified QRTE (64) has been adapted to laser radiation in a cavity with one perfectly reflecting mirror $\left(r_{1}=1-t_{1}=1\right)$, assuming Doppler broadening for the complex line shape function. The calculation is similar to that neglecting coherence and it leads to a transcendental equation for $\delta$, formally similar to Eq. (71), with the substitution $\gamma \leftrightarrow \gamma-g_{0} \lambda_{c} / \sqrt{\pi}$ in the numerator in front of the logarithm. Figure 9 shows the oscillator extraction efficiency $\zeta=I_{\text {out }} /\left(I_{S} g_{0} L\right)$ in terms of the output coupling $t_{2}=1-r_{2}$, for $g_{0} L=2$ and $\alpha L=0.1$, assuming a Doppler coherence length of $0.9 / g_{0}$. The result obtained neglecting coherence is also plotted. The curves show a significant deviation, in particular for large transmission coefficient. Such a deviation suggests that an experimental validation of the QRTE could be obtained from an analysis of the output of a laser with large coherence length (such as He-Ne, e.g. [66]). 


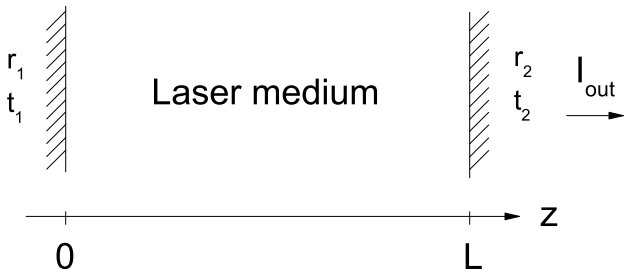

FIG. 8: Schematic representation of a laser cavity. $r_{1,2}$ and $t_{1,2}$ denote the reflection and transmission coefficients of the mirrors.

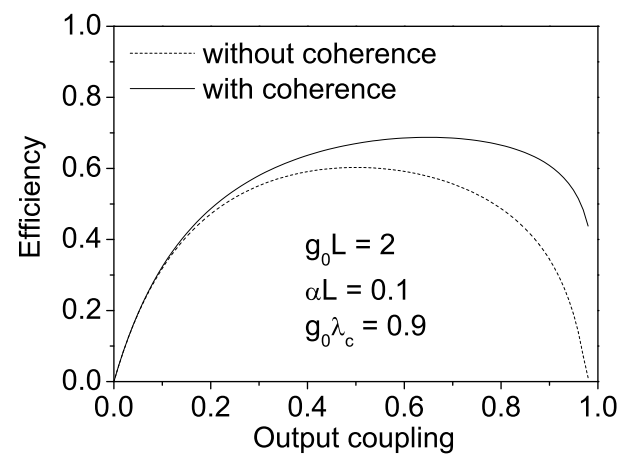

FIG. 9: Plot of the oscillator extraction efficiency in terms of the output coupling $t_{2}=1-r_{2}$. Significant deviations are present if the coherence is retained.

\section{CONCLUSION}

The standard radiative transfer theory assumes large spectral band radiation, in such a way that the energy quanta exchanged between emitters and absorbers are localized in space. In this work, we have addressed the limitations of this assumption in the case of line radiation, using a quantum transport model based on the Wigner phase space formalism. This approach has the advantage of describing the wave-particle duality in a consistent way. The radiation is partially coherent when the characteristic length $\lambda_{c} \sim \lambda^{2} / \Delta \lambda$ is significant with respect to the spatial scales of interest of the system under consideration. This includes the inverse absorption coefficient, commonly referred to as photon mean free path in the standard radiative transfer literature. From the particle point of view, radiation coherence implies photons with finite thermal de Broglie wavelength, interacting nonlocally with massive particles. The description of such non- local interactions amounts to write down and solve an integro-differential equation for the radiation. Because of the Heisenberg uncertainty relations, the photon Wigner function can take negative values and, hence, is not directly interpretable as a probability density function. We have shown that the modeling of observable quantities requires the evaluation of the response of appropriate detector functions. Relevant detector models include phase space coarse-graining at volume larger than $\hbar^{3}$, and also more physical concepts such as photodetection signals. The formalism developed in this work can be applied either to the description of the radiation field or to the kinetics of atomic populations. With applications to ideal cases, we have shown that both are sensitive to coherence effects. The present investigation suggests a refinement of the current radiative transfer models and can find applications to any domain relevant to plasma spectroscopy. This includes astrophysics, inertial and magnetic fusion research, laboratory experiments and technical applications. This also concerns laser physics. In this context, calculations have shown that the output power of a laser is affected by the radiation coherence, suggesting an experimental test of the theory. In its present form, the transport model provides a challenging numerical issue given the nonlocal structure of the quantum radiative transfer equation. A complement to this work should be devoted to a simplified treatment of the problem, e.g., based on the Moyal product series expansion. Another issue concerns the description of partial redistribution in radiation scattering, neglected in this investigation, and could involve master equations similar to those considered in [49]. The formalism presented in this work has been developed with a special emphasis on spatial coherence. A complementary investigation should address temporal coherence on an equal footing, e.g., using generalized Wigner functions defined as both space and time integrals (e.g. [70]).

\section{Acknowledgments}

This work was carried out within the framework of the European Fusion Development Agreement and the French Research Federation for Fusion Studies. It is supported by the European Communities under the contract of Association between Euratom and CEA. The views and opinions expressed herein do not necessarily reflect those of the European Commission.
[1] D. Mihalas, Stellar Atmospheres (W. H. Freeman and Company, San Francisco, 1978).

[2] G. C. Pomraning, The Equations of Radiation Hydrodynamics (Pergamon, Oxford, 1973).

[3] J. E. Bailey, G. A. Rochau, R. C. Mancini, C. A. Iglesias, J. J. MacFarlane, I. E. Golovkin, C. Blancard, Ph. Cosse, and G. Faussurier, Phys. Plasmas 16, 058101 (2009).

[4] B. A. Remington, R. P. Drake, and D. D. Ryutov, Rev. Mod. Phys. 78, 755 (2006).

[5] S. J. Rose, Plasma Phys. Control. Fusion 47, B735 (2005).

[6] F. J. Rogers and C. A. Iglesias, Science 263, 50 (1994). 
[7] T. C. Sangster et al., Nucl. Fusion 47, S686 (2007).

[8] A. Loarte et al., Nucl. Fusion 47, S203 (2007).

[9] D. Reiter, S. Wiesen, and M. Born, Plasma Phys. Control. Fusion 44, 1723 (2002).

[10] V. Kotov, D. Reiter, A. S. Kukushkin, H. D. Pacher, P. Börner, and S. Wiesen, Contib. Plasma Phys. 46, 635 (2006).

[11] M. L. Adams and H. A. Scott, Contrib. Plasma Phys. 44, $262(2004)$.

[12] J. Rosato, V. Kotov, and D. Reiter, J. Phys. B: At. Mol. Opt. Phys. 43, 144024 (2010).

[13] M. Goto, R. Sakamoto, and S. Morita, Plasma Phys. Control. Fusion 49, 1163 (2007).

[14] V. S. Lisitsa, E. E. Mukhin, M. B. Kadomtsev, A. B. Kukushkin, A. S. Kukushkin, G. S. Kurskiev, M. G. Levashova, and S. Yu. Tolstyakov, Plasma Phys. Reports 38, 138 (2012).

[15] J. T. Mendonça and R. Kaiser, Phys. Rev. Lett. 108, 033001 (2012)

[16] G. G. Lister, J. E. Lawler, W. P. Lapatovich, and V. A. Godyak, Rev. Mod. Phys. 76, 541 (2004).

[17] A. Mandelis and C. Feng, Phys. Rev. E 65, 021909 (2002).

[18] A. Liemert and A. Kienle, Phys. Rev. E 86, 036603 (2012).

[19] M. Born and E. Wolf, Principles of Optics: Electromagnetic Theory of Propagation, Interference and Diffraction of Light (Pergamon Press, Oxford, 1964).

[20] M. H. L. Pryce, Proc. R. Soc. London, Ser. A 195, 62 (1948).

[21] T. D. Newton and E. P. Wigner, Rev. Mod. Phys. 21, 400 (1949).

[22] D. Rosewarne and S. Sarkar, Quantum Opt. 4, 405 (1992).

[23] I. Bialynicki-Birula, Progress in Optics (Elsevier, Amsterdam, 1996), vol. XXXVI, p. 245.

[24] M. Hawton and W. E. Baylis, Phys. Rev. A 64, 012101 (2001).

[25] I. Bialynicki-Birula and Z. Bialynicka-Birula, Phys. Rev. Lett. 108, 140401 (2012).

[26] I. Bialynicki-Birula and Z. Bialynicka-Birula, Phys. Rev. A 86, 022118 (2012).

[27] Z.-Y. Wang, C.-D. Xiong, and Q. Qiu, Phys. Rev. Lett. 109, 188901 (2012).

[28] I. Bialynicki-Birula and Z. Bialynicka-Birula, Phys. Rev. Lett. 109, 188902 (2012).

[29] R. K. Osborn and E. H. Klevans, Ann. Phys. (N.Y.) 15, 105 (1961).

[30] W. E. Brittin and W. R. Chappell, Rev. Mod. Phys. 34, 620 (1962).

[31] R. J. Gelinas and R. L. Ott, Ann. Phys. (N.Y.) 59, 323 (1970).

[32] J. Cooper and P. Zoller, Astrophys. J. 277, 813 (1984).

[33] F. R. Graziani, J. Quant. Spectrosc. Radiat. Transfer 83, 711 (2004).

[34] R. M. G. M. Trines, R. Bingham, L. O. Silva, J. T. Mendonça, P. K. Shukla, C. D. Murphy, M. W. Dunlop, J. A. Davies, R. Bamford, A. Vaivads, et al., Phys. Plasmas 16, 055904 (2009).

[35] E. Wigner, Phys. Rev. 40, 749 (1932).

[36] P. Carruthers and F. Zachariasen, Rev. Mod. Phys. 55, 245 (1983).

[37] J. Rammer and H. Smith, Rev. Mod. Phys. 58, 323 (1986).
[38] R. Balescu, Equilibrium and Nonequilibrium Statistical Mechanics (Wiley-Interscience, New York, 1975).

[39] D. F. Styer, M. S. Balkin, K. M. Becker, M. R. Burns, C. E. Dudley, S. T. Forth, J. S. Gaumer, M. A. Kramer, D. C. Oertel, L. H. Park, et al., Am. J. Phys. 70, 288 (2002).

[40] K. Zhang, P. Meystre, and W. Zhang, Phys. Rev. Lett. 108, 240405 (2012).

[41] A. Ferraro and M. G. A. Paris, Phys. Rev. Lett. 108, 260403 (2012).

[42] M. A. Alonso, Adv. Opt. Phot. 3, 272 (2011).

[43] J. Rosato, Am. J. Phys. 78, 851 (2010).

[44] J. Rosato, Phys. Rev. Lett. 107, 205001 (2011).

[45] J. Rosato, Transport Theor. Stat. Phys. 41, 214 (2012).

[46] C. Cohen-Tannoudji, J. Dupont-Roc, and G. Grynberg, Photons and Atoms: Introduction to Quantum Electrodynamics (Wiley-Interscience, New York, 1989).

[47] C. Cohen-Tannoudji, J. Dupont-Roc, and G. Grynberg, Atom-Photon Interactions: Basic Processes and Applications (Wiley-Interscience, New York, 1992).

[48] E. L. Degl'Innocenti, Sol. Phys. 85, 3 (1983).

[49] V. Bommier, Astron. Astrophys. 328, 706 (1997).

[50] H.-P. Breuer and F. Petruccione, The Thoery of Open Quantum Systems (Oxford University Press, New York, 2002).

[51] Iu. L. Klimontovich, Sov. Phys. JETP 6, 753 (1958).

[52] C. Zachos, D. Fairlie, and T. Curtright, Quantum Mechanics in Phase Space (World Scientific, Singapore, 2005).

[53] H.-W. Lee, Phys. Rep. 259, 147 (1995).

[54] D. Reiter, M. Baelmans, and P. Börner, Fus. Sci. Technol. 47, $172(2005)$.

[55] J. Spanier and E. M. Gelbard, Monte Carlo Principles and Neutron Transport Problems (Dover Publications, Mineola, 2008)

[56] T. Holstein, Phys. Rev. 72, 1212 (1947).

[57] L. M. Biberman, Zh. Eksp. Teor. Fiz. 17, 416 (1947).

[58] F. E. Irons, J. Quant. Spectrosc. Radiat. Transfer 22, 1 (1979).

[59] F. B. Rosmej, V. S. Lisitsa, and R. Stamm, Europhys. Lett. 73, 342 (2006).

[60] A. P. Kazantsev, V. S. Smirnov, and V. P. Yakovlev, Sov. Phys. JETP 55, 1004 (1982).

[61] M. Abramowitz and I. A. Stegun, Handbook of Mathematical Functions with Formulas, Graphs, and Mathematical Tables (Department of Commerce, Washington DC, 1964).

[62] N. C. Dias and J. N. Prata, J. Math. Phys. 43, 4602 (2002).

[63] D. Taj, L. Genovese, and F. Rossi, Europhys. Lett. 74, 1060 (2006).

[64] E. Stambulchik, S. Alexiou, H. R. Griem, and P. C. Kepple, Phys. Rev. E 75, 016401 (2007).

[65] J. Rosato, H. Capes, and R. Stamm, Phys. Rev. E 86 , 046407 (2012).

[66] P. W. Milonni and J. H. Eberly, Lasers (Wiley Interscience, New York, 1988).

[67] P. Laures, Phys. Lett. 10, 61 (1964).

[68] W. W. Rigrod, J. Appl. Phys. 36, 2487 (1965).

[69] J. K. Rice, G. C. Tisone, and E. L. Patterson, IEEE J. Quant. Electron. QE-16, 1315 (1980).

[70] J. T. Mendonça and A. Serbeto, Phys. Plasmas 13, 102109 (2006). 